\title{
RiESGO DE CONSUMO DE CIGARRILLO EN POBLACIÓN ADULTA COLOMBIANA
}

\section{Risk of Cigarette Consumption in Colombian Adult Population}

\author{
Constanza Londoño Pérez ${ }^{1}$ y Laura Viviana Gómez ${ }^{2}$ \\ https://doi.org/10.17533/udea.rp.v12n1a02
}

\section{Resumen}

El objetivo del presente estudio fue describir condiciones de incidencia, prevalencia, severidad de consumo de cigarrillo y factores psicosociales de riesgo (expectativas, motivación-tentaciones, identidad de consumo, ambiente de consumo y variables sociodemográficas) del consumo de cigarrillo. La muestra final estratificada no aleatorizada de 1364 participantes incluyó 569 hombres $(41,7 \%)$ y 795 mujeres $(58,3 \%)$, con edades entre 18 y 84 ańos $(M=28,33$ y $\mathrm{DE}=10,31)$, provenientes de 14 Departamentos de Colombia. Para identificar los factores de riesgo de consumo de tabaco se realizó un análisis con Mode-

\section{Abstract}

The objective of the present study was to describe conditions of incidence, prevalence, severity of cigarette consumption and psychosocial risk factors (expectations, motivation-temptations, identity of consumption, environment of consumption and sociode-

Recibido: 2020-04-21 / Aceptado: 2020-05-28

Para citar este artículo en APA: Londońo, C. y Gómez, L. (2020). Riesgo de consumo de cigarrillo en población adulta colombiana. Revista de Psicología Universidad de Antioquia, 12(1), 25-46. doi: https://doi.org/10.17533/udea.rp.v12n1a02 los de Ecuaciones Estructurales (SEM) con el programa Amos. Los resultados indican que el 30,3\% eran fumadores, la mayoría con consumo alto o dependiente. Así mismo, la alta identidad de consumo, las expectativas positivas sobre los efectos de la sustancia y la alta motivación-tentación pueden ser considerados factores de riesgo ya que se asocian al mantenimiento del consumo y al fracaso en los intentos de cesación.

Palabras clave: consumo de tabaco, factores de riesgo, adultos jóvenes, identidad personal, creencias.

mographic variables) of cigarette consumption. The final non-randomized stratified sample of 1,364 participants included 569 men $(41,7 \%)$ and 795 women $(58,3 \%)$, with ages between 18 and 84 years old $(\mathrm{M}=28,33$ and DT $=10,31)$, from 14 Departments of

Universidad Católica de Colombia, Bogotá. Correo: clondono@ucatolica.edu.co; https:// orcid.org/0000-0003-3273-3658

2 Universidad Católica de Colombia, Bogotá. Correo: lvgomezv@gmail.com; https://orcid. org/0000-0002-7973-5848 
Colombia. To identify the risk factors for tobacco consumption, an analysis was carried out with Structural Equation Models (SEM) with the AMOs software. The results indicate that $30,3 \%$ were smokers, mostly with high or dependent consumption. Likewise, the high identity of consumption, the positive expectations about the effects of the substance and the high motivation of temptation can be considered risk factors since they are associated with the maintenance of consumption and the failure of cessation attempts.

Keywords: tobacco consumption, risk factors, young adults, personal identity, expectations, belief.

\section{Introducción}

El consumo de cigarrillo es un problema de salud pública relacionado con más del $70 \%$ de las muertes por cáncer y se predice que, si continúa el aumento progresivo observado hasta ahora, en el 2030 más de 8 millones de fumadores morirán por esta causa. El consumo de cigarrillo, para esta organización, se relaciona con la disminución de la calidad de vida, reducción en la productividad laboral y social y aumento en el gasto sanitario (Organización Panamericana de la Salud [ops], 2014); incluso, se ha aumentado debido a los estilos de vida que incrementan el riesgo epidemiológico de la población joven y adulta (Organización Mundial de la Salud [OMs], 2020).

Para Becońa (2002) la prevención del consumo de drogas es insuficiente cuando se dirige únicamente a la reducción de la oferta de la sustancia, por tanto, es necesario realizar control de la demanda centrado en las personas que implique la modificación de actitudes, creencias y factores de riesgo; además, los profesionales a cargo de estos procesos requieren un grado avanzado de conocimiento acerca de las formas de consumo y los tipos de consumidores para facilitar la prevención del inicio y el acompañamiento efectivo en procesos de abandono de consumo.

Múltiples factores han sido asociados al inicio y mantenimiento del consumo de tabaco, entre ellos se encuentran los factores ambientales que promueven el uso, a pesar del diseño e implementación de factores de control como la restricción legal de consumo en lugares públicos y de la venta a menores. Colombia aún cuenta con un fácil acceso y disponibilidad como resultado de un bajo costo (Departamento Administrativo Nacional de Estadística [DANE], 2016), de la venta libre y poco regulada en lugares ubicados 
cerca de las viviendas, lugares de trabajo y entidades educativas (Comunidad Andina, 2013; Gobierno Nacional de la República de Colombia, 2014; Ortiz y Rodríguez, 2009) así como de la publicidad que alude emociones positivas derivadas del consumo.

Los significados sociales conferidos al tabaco que incluyen normas, reglas, rituales de consumo son factores ambientales determinantes (Novoa-Gómez, Barreto y Silva, 2012; Villena et al., 2009). Fumar se ha asociado al consumo de bebidas alcohólicas, cafeína, drogas ilegales, descanso, manejo de estrés (Campo-Arias y Díaz-Martínez, 2006; Cogollo y Gómez, 2013) y al intercambio social efectivo (Ávila, 2003; Becońa et al., 2011; Becoña y Vázquez, 1997). Al respecto, la exposición a modelos permisivos, la aceptación social de consumo (Li, Mao, Stanton y Qun, 2010) y la presión de pares (DeLay, Kiuru, Salmela-Aro, y Nurmi, 2013) facilitan el consumo de tabaco.

Dichos factores ambientales, sociales y culturales suman su acción a características individuales que han sido identificadas como facilitadoras del consumo de tabaco; entre otras, las creencias positivas hacia los efectos del cigarrillo sobre el control de estrés, la reducción de peso y el manejo de la ansiedad (Baha y Le Faou, 2013; Bautista, Gómez, Aguilar, Herrador y Alfaro, 2016; Hanson, 2014) y la probabilidad percibida acerca del riesgo (Calleja, 2012; Halterman, Borrelli, Conn, Tremblay y Blaakman, 2010).

Así mismo, los factores psicológicos como las expectativas de alivio del estrés derivadas de la idea de que fumar facilita la regulación del afecto, ayuda a controlar las situaciones negativas y facilita el intercambio social, combinadas con un ambiente social de consumo (cercanía con otros fumadores en ambientes que otorgan una valencia positiva al fumar), hacen que la persona se identifique como fumadora, refuerce comportamientos asociados al consumo y perciba el abandono o disminución del consumo poco factible (Brown, Carpenter y Sutfin, 2011; Haight, Dickter y Forestell, 2012; Novoa-Gómez et al., 2012; Rodríguez y Londoño, 2010; Rubinstein, Rait, Sen y Shiffman, 2014; Velasco, Londoño, Forero, Romero y Ruiz, 2017).

Lujic, Reuter y Netter (2005) y Londoño, Cabarcas y Salamanca (2020) coinciden al indicar que los fumadores tienen al menos siete tipos de moti- 
vaciones para consumir: 1) motivaciones sociales, centradas en la aceptación del consumo por parte de pares y familia; 2) motivaciones sensoriales, experimentadas al fumar (tacto, sabor y olor); 3) motivación por el refuerzo dopaminérgico, relacionado con el placer que genera la nicotina; 4) motivación para estimulación, reflejada al utilizar el consumo de cigarrillo para incrementar la atención ante actividades cotidianas o de resolución de conflictos; 5) motivación de alivio de estados de ánimo negativos; 6) motivación de dependencia, derivada de los síntomas de abstinencia; y 7) motivación automática o habitual, que no se explica por dependencia.

Aproximadamente el $90 \%$ de los fumadores empiezan a fumar en la adolescencia antes de la mayoría de edad y esto hace que se instaure con mayor facilidad el hábito de consumo (American Cancer Society [ACs], 2014; Borracci y Mulassi, 2015). La prevalencia de consumo es mayor en hombres que en mujeres, aunque en los últimos años las cifras muestran una reducción de la diferencia debido al incremento significativo del consumo en mujeres (OPS, 2010; Centro para el Control y la Prevención de Enfermedades [CDC], 2014).

La investigación acerca del consumo de tabaco y los factores relacionados con su ocurrencia es abundante; no obstante, el conocimiento acerca de las características de cada uno de los tipos de fumadores existentes es limitado. Por ejemplo, quienes consumen a diario responden a estímulos condicionados - como ver una taza de café, esperar, sentir frío o calor, el olor del cigarrillo, la acumulación de trabajo y la asistencia a reuniones sociales- a diferencia de aquellos fumadores ocasionales que sienten deseos de fumar únicamente ante señales asociadas directamente con el cigarrillo (Haight, Dickter y Forestell, 2012; Novoa-Gómez et al., 2012). El objetivo del presente estudio fue describir condiciones de incidencia, prevalencia, severidad de consumo de cigarrillo y los factores psicosociales de riesgo (expectativas, motivación-tentaciones, identidad de consumo, ambiente de consumo y variables sociodemográficas) del consumo de cigarrillo. 


\section{Metodología}

\section{Tipo de estudio}

El presente es un estudio prospectivo descriptivo correlacional con análisis multivariante.

\section{Participantes}

La muestra no aleatorizada estratificada estuvo conformada por 1364 personas entre 18 y 84 ańos de edad (M[media]= 28,33; DE [desviación estándar]= $10,31)$; 569 hombres $(41,7 \%)$ y 795 mujeres $(58,3 \%)$, principalmente jóvenes ( $49,6 \%$ de 22 a 35 años) y adolescentes mayores (30,9\% de 18 a 21 años), de estratos socioeconómicos medio bajos (2) y medios (3) (DANE, 2020). Los criterios de inclusión básicos fueron ser colombiano mayor de edad y residir en el país.

\section{Instrumentos}

Cuestionario para la Clasificación de Consumidores de Cigarrillo C4: este instrumento elaborado por Londoño y Rodríguez (2007) se aplica con el fin de evaluar la severidad de consumo de cigarrillo (nivel de consumo) teniendo en cuenta la frecuencia, intensidad y factores asociados al consumo. Cuenta con 15 ítems que ofrecen opciones de respuesta diversas para cada pregunta: el puntaje va de 0 a 30, y los rangos de puntaje están de acuerdo con los niveles de consumo; en el dependiente el puntaje es de 19 a 30, consumidor fuerte es de 5 a 8 puntos, consumidor moderado de 9 a 18 puntos, no consumidor puntaje de 0 . En cuanto a los indicadores de calidad psicométrica, este instrumento obtuvo un Alfa de Cronbach de 0,90 y los ítems cuentan con un puntaje entre 1 y 1,3 en el infit, y puntajes menores a 2,0 en el outfit; por ello, se considera que todos los ítems están correctamente ajustados al modelo de Rasch.

Cuestionario de Expectativas hacia el Cigarrillo: es un cuestionario que mide las creencias y expectativas positivas frente al consumo de cigarrillo elaborado por Londoño y Rodríguez (2007) y validado por Londoño, Velasco y Gómez 
(2019). Tiene 23 ítems que indagan por indicadores de efectos fisiológicos evaluados $(7,8,9,10,12,13,16)$, imagen social $(18,19,21,23)$, expectativas de relajación $(4,17$ y 22), interacción social $(2,3,14,15)$ y expectativas en general $(1,5,6,20)$. La opción de respuesta es falso/verdadero y se obtiene un valor de 1 para verdadero y de 0 para falso, por lo que la calificación tiene un rango entre 0 y 23. El nivel de confiabilidad es alto (Alfa de Cronbach de $0,92)$, los ítems cuentan con puntajes entre 1 y 1,3 el infit y puntajes menores a 2,0 en el outfit y se ajustan al modelo de Rasch (Rodríguez y Londońo, 2010; Castellanos y Londońo, 2014).

Cuestionario de Motivación-Tentación: este instrumento diseñado por Londoño y Rodríguez (2007) y revalidado por Castellanos y Londoño (2014) mide los factores facilitadores del consumo de cigarrillo como estímulos ambientales que incitan a las personas a fumar; cuenta con 13 ítems cuyas opciones de respuesta son presentadas en una escala tipo Likert con un puntaje de 1 a 5 según el grado de tentación y la calificación se encuentra entre 13 a 65. Los puntajes altos indican que la persona se siente muy tentada a fumar y permite identificar una lista de los estímulos desencadenantes de la conducta. La escala tiene un nivel alto de confiabilidad, los ítems cuentan con puntajes entre 1 y 1,3 el infit y puntajes menores a 2,0 en el outfit y se ajustan al modelo de Rasch (Alfa de Cronbach 0,95).

Cuestionario de Identidad de Consumo (CICC): este instrumento elaborado por Castellanos y Londoño (2014) mide la identificación del consumo de cigarrillo por medio de los factores asociados a la percepción, expectativas, valoraciones, atribuciones y riesgo percibido del consumo de cigarrillo; consta de 41 ítems que alternan opciones de respuesta de falso/verdadero y escalas tipo Likert según el indicador evaluado. Cuenta con un alto nivel de confiabilidad reflejado en el Alfa de Cronbach de 0,88.

\section{Procedimiento}

Inicialmente se contó con la firma del consentimiento informado, para proceder con la aplicación de los instrumentos mencionados anteriormente. Luego se analizaron los datos para conocer las características psicológicas de la muestra evaluada. 


\section{Análisis de Resultados}

Para determinar la asociación predictiva de riesgo entre las variables de estudio (variables explicativas) y la conducta de fumar (variable de respuesta) se usó un modelo de ecuaciones estructurales, por medio del programa estadístico IBM SPSS AMOS (versión 23,0). Este modelo permite analizar los factores asociados por medio de la evaluación de las medidas incrementales de ajuste que permiten indicar el grado de ajuste entre el modelo teórico y el modelo empírico resultante, se realiza a partir de las siguientes medidas que se encuentran en el rango de cero (mal ajuste) y uno (ajuste perfecto); raíz cuadrada del error cuadrático medio RMSEA (Root Mean Square Error of Approximation), el índice de ajuste normalizado o NFI (Normed Fit Index), el índice comparativo de ajuste o CFI (Comparative Fit Index), y por último la medida de chi cuadrado que refleja la existencia de diferencias inesperadas entre el modelo teórico propuesto y el modelo empírico, por tanto se espera que el chi no sea significativo, es decir, que el valor de p debe ser mayor a 0,05 y que el chi se aproxime a 0 (Bisquerra, 1989). Finalmente se reportan los índices corregidos usando el método de máxima verosimilitud, mínimos cuadrados generalizados y la estimación por distribución libre de acuerdo con el tamaño de la muestra para evitar sesgos.

\section{Resultados}

Respecto a la clasificación y descripción de consumo de cigarrillo, aproximadamente la mitad de los fumadores reportaron consumo fuerte $(49,2 \%)$ y en su mayoría eran hombres $(51,8 \%)$; cerca de la tercera parte $(34,9 \%)$ reportaron consumo dependiente, principalmente hombres aunque con un número importante de casos en mujeres; seguido del consumo moderado de riesgo $(12,8 \%) y$, en menor medida, del consumo leve $(2,2 \%)$, niveles donde hombres y mujeres presentaron proporciones semejantes de consumo. El 65,5\% llevaba fumando menos de un año y el 24,4\% entre 3 y 5 años. El 6,1\% informaron encontrarse en proceso de cesación y cerca del $70 \%$ de fumadores expresó deseo de abandonar el consumo y reportó haber intentado la cesación en múltiples ocasiones sin éxito. 


\section{Figura 1}

Distribución del consumo de tabaco

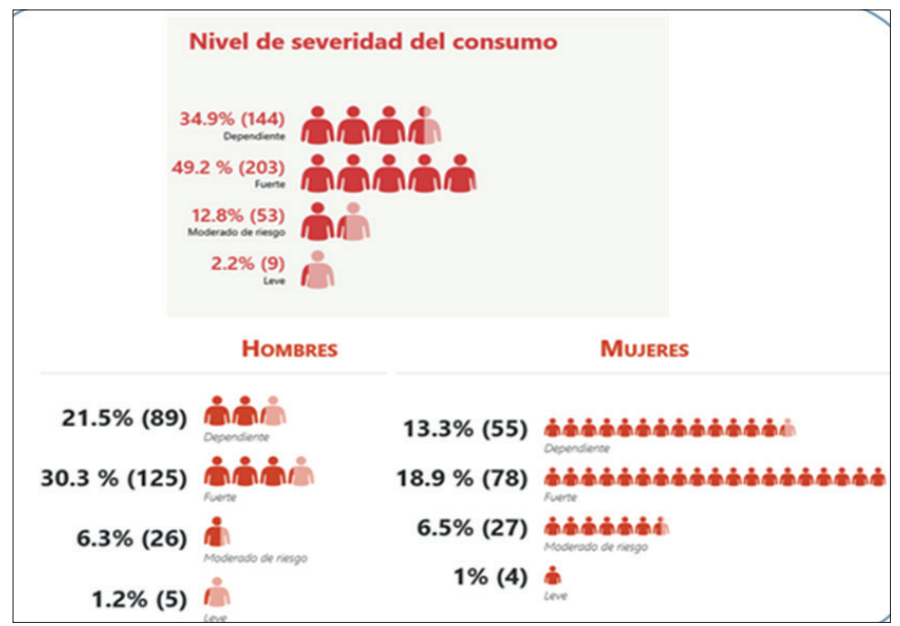

Nota. Elaboración propia.

Frente a la exposición al humo, se observó que casi la totalidad de la muestra fumaba simultáneamente con otras personas, es decir, tenían doble exposición al humo de tabaco $(95,2 \%)$; a más de la mitad no le incomodaba el humo de otros fumadores aun cuando ellos no estuviesen fumando, por lo que puede afirmarse que además eran fumadores pasivos (66,3\%); aproximadamente a la cuarta parte de fumadores les molestaba el humo pero permanecían en el lugar de exposición (22\%) y un número reducido prefería evitar exponerse al humo de otros fumadores (11,6\%).

A partir de los resultados arrojados de la aplicación de los instrumentos (i.e. C4, Cuestionario de Expectativas hacia el Cigarrillo, Cuestionario de Motivación-Tentación y cicc) se encontró que, entre los factores asociados al consumo, hay un alto grado de motivación-tentación sentido ante estímulos discriminativos que generaban el deseo de consumir ( $\mathrm{M}=40$ puntos en el grado de tentación experimentado) especialmente desencadenado por estímulos sociales (una fiesta o reunión), por la sensación de necesidad de fumar, por situaciones estresantes que generan emociones negativas (Preocupación /depresión) y por el simple deseo de fumar (Tabla 1). 


\section{Tabla 1}

Factores de riesgo asociados al consumo de cigarrillo

\begin{tabular}{lcc}
\hline \multicolumn{1}{c}{ Factor } & $\mathrm{M}$ & $\mathrm{DE}$ \\
\hline Motivación & 40,1 & 13,5 \\
Situaciones sociales (Fiestas / reuniones) & 3,5 & 1,4 \\
Alivio de emociones negativas (Preocupación /depresión) & 3,4 & 1,5 \\
Deseos de fumar & 3,6 & 1,4 \\
Necesidad de fumar & 3,5 & 1,5 \\
& & \\
Expectativas positivas sobre efecto de la nicotina & 7,1 & 5,1 \\
Manejo del estrés (Efectos fisiológicos positivos/relajación) & 3,0 & 2,2 \\
Mejoramiento imagen social e interacción social & 0,7 & 1,1 \\
& & \\
Expresiones de la identidad de consumo & 46,3 & 14,4 \\
Inhalar profundo & 1,6 & 0,7 \\
Tener afectada la salud & 1,8 & 0,6 \\
Disfrutar fumar & 1,6 & 0,7 \\
Aceptación por parte del círculo social al que pertenece & 1,7 & 0,7 \\
Fumar habitualmente & 1,6 & 0,7 \\
\hline
\end{tabular}

${ }^{*} \mathrm{M}=$ media $\quad \mathrm{DE}=$ desviación estándar

\section{Expectativas positivas sobre los efectos del consumo}

Se evidencia la existencia de altas expectativas positivas acerca de los efectos del consumo esperados: la generación de reacciones fisiológicas positivas/ agradables como la relajación ( $\mathrm{M}=3,0$ puntos), el mejoramiento de la imagen social o la interacción social ( $\mathrm{M}=0,7$ puntos) (ver Tabla 1 ).

\section{Identidad de consumo}

Los fumadores participantes reportan tener alta identidad de consumo ( $M=$ 46,3 puntos) expresada principalmente en la aparición de señales de afecta- 
ción de la salud ( $\mathrm{M}=1,8$ puntos), de la expresión de aceptación de la conducta de fumar por parte del círculo social ( $\mathrm{M}=1,7$ puntos), de hecho, de inhalar profundo, disfrutar fumar y hacerlo de forma habitual ( $\mathrm{M}=1,6$ puntos respectivamente) (ver Tabla 1).

\section{Correlaciones}

Los resultados muestran que existe correlación directa y significativa (con un $p$ menor o igual a ,05) entre la identidad de consumo, el grado de motivación-tentaciones experimentado ante situaciones disparadoras del consumo y las expectativas positivas acerca de los efectos de la sustancia con nivel de consumo (ver Tabla 2).

Tabla 2

Análisis de correlaciones de las variables asociadas el consumo de cigarrillo

\begin{tabular}{ccccc}
\hline & NC & MT & Ex & IC \\
\hline IC & 1 &, $387^{* *}$ &, $364^{* *}$ &, $493^{* *}$ \\
MT & 1 &, $522^{* *}$ &, $545^{* *}$ \\
Ex & & & 1 &, $495^{* *}$ \\
IC & & & & 1 \\
\hline
\end{tabular}

Nota . NC=nivel consumo, MT=motivación-tentaciones, Ex=Expectativas, IC=Identidad de Consumo. $p={ }^{*}:, 05,{ }^{* *}, 01$

\section{Modelo Predictivo: factores psicosociales de riesgo}

El modelo predictivo de consumo de cigarrillo derivado del análisis realizado con ecuaciones estructurales indicó que la interacción de variables como la edad, el sexo, las expectativas positivas sobre los efectos del cigarrillo, el grado de motivación tentación experimentado hacia el consumo de cigarrillo y la identidad de consumo explican el $56 \%$ de la varianza de la conducta de fumar, esta última entendida como la variable dependiente. Las variables que se relacionan de forma directa con una alta probabilidad de emitir esta conducta 
son la alta identidad de consumo $(\mathrm{PRVE}=, 48)$, la alta motivación tentación sentida (PRVE $=, 23$ ) en especial en jóvenes más cercanos al límite superior de edad ya que la relacionan con independencia $(\mathrm{PRVE}=, 13)$. De otra parte, existen variables como las expectativas ( $\mathrm{PRVE}=, 37)$ y el hecho de ser hombre $(\mathrm{PRVE}=, 28)$ que predicen el grado de motivación tentaciones experimentado por el fumador (Figura 1).

\section{Figura 2}

Modelo Predictivo de consumo de cigarrillo

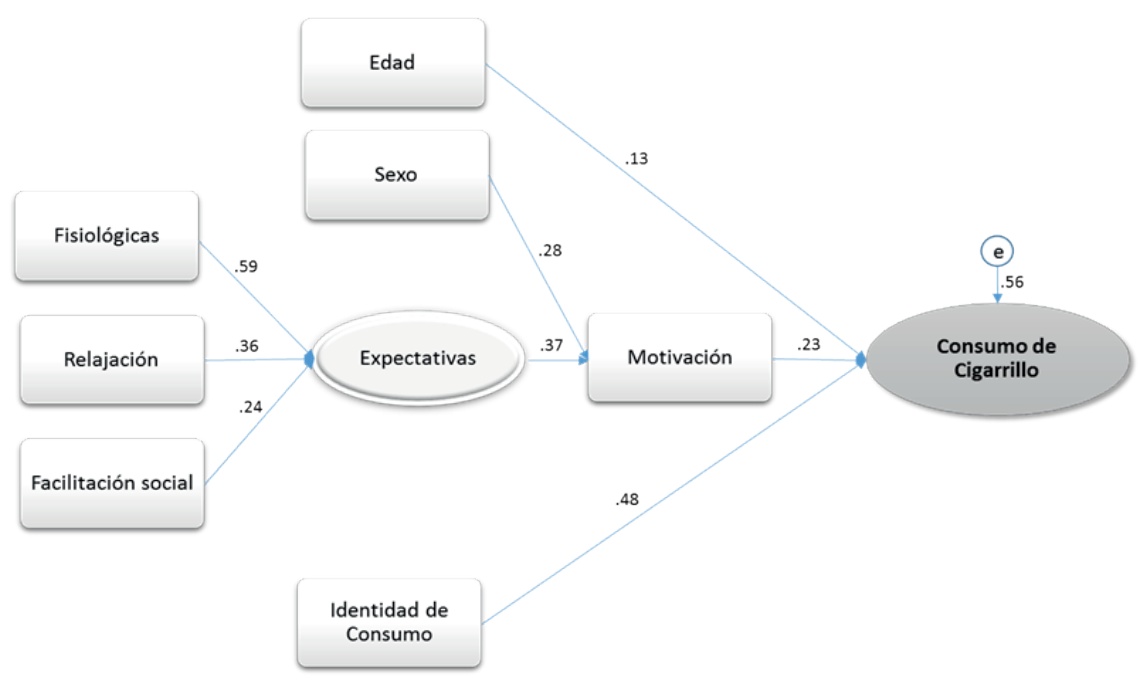

Nota. Elaboración propia.

Al calcular los índices corregidos para el tamaño de la muestra, se encontró que todos denotan que el modelo tiene un nivel de ajuste adecuado, el RMSEA es inferior a 0,04, el NFI es mayor a 0,80, el CFI es superior a 0,95 y el chi cuadrado de bondad del ajuste tiene una significancia superior a 0,05 , es decir, el modelo cumple las condiciones estadísticas esperadas y es recursivo porque no tiene efectos circulares o recíprocos entre sus variables. 
Tabla 3

Índices de ajuste del modelo de ecuaciones estructurales identificado consumo de cigarrillo

\begin{tabular}{lccc}
\hline Índices de ajuste & & Resultado & Indicador \\
\hline $\begin{array}{l}\text { Raíz cuadrada del error cuadrático } \\
\text { medio }\end{array}$ & RMSEA &, 00 & --- \\
Índice de ajuste normalizado & NFI &, 90 & -- \\
Índice comparativo de ajuste & CFI & 1,0 & -- \\
Chi cuadrado & Chi &, 00 & $p$ mayor a \\
& & 4,05 & --- \\
Grados de libertad & $\mathrm{Gl}$ & 4,05 & $p$ menor a \\
Varianza explicada & $\mathrm{R}^{2}$ &, 56 & 0.05 \\
\hline
\end{tabular}

Nota. $p=$ probabilidad de error.

\section{Discusión}

Respecto a los factores de riesgo relevantes, se encontró que los fumadores estaban expuestos constantemente al propio humo y al de otros consumidores, y este ambiente de consumo interactuaba como factor predictor sumado a los factores de riesgo que se describen a continuación. En cuanto a las expectativas, las que mayor valencia tenían en los fumadores fueron las relacionadas con los efectos fisiológicos esperados de descanso, disminución de la tensión muscular y mejora del estado de ánimo, especialmente expresadas por los fumadores dependientes y de consumo fuerte; entre las de menor valencia aparecen las expectativas relacionadas con aspectos sociales como la mejora de la imagen social y proyección de seguridad especialmente reflejadas en los consumidores moderados y ocasionales, por lo que el consumo de cigarrillo se ha asociado a consumo de alcohol y esto podría verse como un factor de riesgo de poli consumo de SPA [Sustancias Psicoactivas] y potenciador de sus efectos.

Se ha descrito el consumo de cigarrillo como estrategia para manejar la ansiedad y el estrés (cf. Bautista et al., 2016; García del Castillo, 2012; Wood, Cano-Vindel, Iruarrizaga, Dongil, Salguero, 2010), y que las personas con consumo moderado o leve fuman por curiosidad o por motivos sociales, 
aunque luego pueda funcionar como precipitante para el desarrollo de la dependencia (Wood et al., 2010). También Ferreira, Sivalli y Baldini (2014) encontraron que el consumo de cigarrillo se asocia a recuerdos positivos en el entorno familiar y social que se trasponen como creencias reforzantes y motivos válidos para consumir. Calleja (2012) encontró que estas expectativas se forman a partir de las creencias culturales, las prácticas sociales de consumo, presión de pares y falta de claridad frente al no consumo; sin embargo, estos factores no fueron evaluados de forma directa en la presente investigación, por lo que se deberá ampliar en futuras investigaciones.

Otro factor predictor del consumo de cigarrillo fue la tentación o motivación a fumar bajo emociones fuertes de componente tanto negativo como positivo; en esta línea, otros autores habían notado que las motivaciones para consumir cigarrillo se relacionan con la evitación de malestar emocional como tristeza, nerviosismo, aburrimiento, cansancio, soledad y estas se mantienen si su entorno cercano y pareja son fumadores (Marqueta et al., 2013), por lo que perciben el cigarrillo como solución a un estado de alteración emocional, pero la relación que se ha encontrado es que los efectos relajantes o de placer que ocasiona el consumo de cigarrillo son ocasionados por la adicción que genera la nicotina y que los estados de ansiedad son por la falta de esta sustancia en el cerebro (Córdoba y Samitier, 2015); el consumo de cigarrillo se mantiene por un refuerzo negativo, por el efecto a nivel cerebral de sus componentes y por motivaciones sociales del consumo, como ambientes facilitadores y la idea persistente de una cultura favorecedora del consumo. De acuerdo con Ávila (2003) estas motivaciones se evidenciaban en fumadores jóvenes, sin embargo, es contradictoria con los resultados encontrados.

Respecto de la severidad de consumo, atípicamente la mayor parte de los fumadores encuestados tenían un nivel de consumo dependiente o fuerte, sin embargo, estos resultados difieren de estudios previos que plantean que la mayor parte de fumadores se concentra en el consumo moderado y fuerte (Gobierno Nacional de la República de Colombia, 2013) o que el consumo ha disminuido (Ministerio de Salud y Protección Social, 2015). Esta diferencia se puede explicar desde varios puntos de vista, probablemente se puedo presentar una autoselección de fumadores (debido a la manera como se denominó la 
encuesta) y ello afecta la posible generalización de los resultados del presente estudio; también se podría sospechar un posible incremento en el consumo de tabaco en la población colombiana, o que la sensibilidad del C4 facilita la identificación de los distintos niveles de consumo tal como lo proponen Londońo et al. (2019), o bien, porque en general la información disponible de estudios epidemiológicos o de tasa y descripción de consumo de cigarrillo no es actualizada (Becońa, 2014).

De otra parte, más de la mitad de los fumadores deseaba dejar de fumar y lo había intentado en promedio 3,61 ocasiones con un límite máximo de 20 veces. Otros estudios habían notado que un alto porcentaje de fumadores desea dejar de fumar y han tenido intentos de cesación porque consideran riesgoso el consumo de tabaco. Es preciso tener en cuenta que cada intento fallido tiene un impacto negativo sobre el control percibido y la posibilidad real de abandono (Álvarez-Aguirre, Alonso-Castillo y Guidorizzi, 2014) que deja como resultado que el fumador se reafirme que no es posible abandonar el consumo y, sumado a esto, es importante notar la falta de programas de acompañamiento en el país, debido a que los programas actuales se han centrado principalmente en una estrategia basada en el control de la venta, más que en el acompañamiento efectivo a los fumadores, y si estos fumadores con deseo de abandono contaran con la intervención de un profesional adecuado podrían llegar a la cesación efectiva (Castañal-Canto et al., 2012; Aveyard y West, 2007).

Igualmente, se evidencia que los fumadores fuertes y dependientes tienen alta identidad de consumo expresada en señales de afectación de la salud, aceptación del consumo por parte del grupo social, aparición de conductas como inhalar profundo y fumar habitualmente. Cabe aclarar que, a pesar de que los fumadores refieren un consumo habitual, la habitualidad no está definida desde el instrumento. La identidad implica un proceso subjetivo de interpretación de la realidad donde se encuentran incorporadas experiencias vitales asociadas al consumo y representadas en creencias, valoraciones y actitudes de sí mismo y del mundo (Castellano y Londońo, 2014; Molano y Montua, 2015). Las afirmaciones hechas por los fumadores en torno a la identidad pueden influir positiva y negativamente en la decisión de cesar 
o disminuir el consumo; por tanto, la interacción contradictoria que se da entre creencias positivas del grupo social en torno a los efectos deseables del consumo de cigarrillo y la recepción de mensajes de prevención que resaltan la importancia de abandonar la sustancia, puede ocasionar que se sientan vulnerados ya que al parecer no es reconocida su independencia y juventud. En esta misma línea Londoño et al., (2019) encontraron que la identidad se relaciona con el nivel de consumo, pues a menor percepción de riesgo hay menor identificación de consumo y por tanto menor deseo de cesación. Sin embargo, este aspecto ha sido poco abordado en las investigaciones y por ello merece mayor exploración.

Por otro lado, se encuentra la exposición al humo: los fumadores, a pesar de que indicaron que preferían ambientes libres de humo, permanecían en sitios en los que había personas fumando o aún se percibía el olor a cigarrillo y se mantenían en contacto con personas que olían a cigarrillo, por lo que estaban en una exposición doble o triple al humo, especialmente los consumidores fuertes y dependientes (relacionado con el fumar como estilo de vida). Estudios previos han mostrado que los fumadores se exponen con mayor frecuencia a ambientes de consumo con humo de segunda y tercera mano, en lugares como carros, cafeterías, lugares de trabajo, discotecas y demás lugares de ocio (Clemente et al., 2012). Ahora bien, se identifica un cambio de rol situacional y un incremento del riesgo de exposición al humo ya que, en ocasiones, eran fumadores activos y, en otras, pasivos.

Es importante anotar que los fumadores reportaron alta exposición al humo de segunda y tercera mano, por lo que es de gran importancia continuar con la implementación de políticas que mitiguen la exposición al humo por sus consecuencias negativas en la salud, considerando que no existe un nivel libre de riesgo de exposición al humo de tabaco (Sandoya y Bianco, 2011); a pesar de que se sentían incómodos cuando alguien desconocido fumaba cerca de ellos, estos no se alejaban del lugar, de modo que estaban expuestos al riesgo derivado del consumo pasivo de cigarrillo. En contraste, casi la totalidad de la muestra de fumadores indicó que acompańaban a alguien conocido a fumar, sin denotar conciencia del riesgo de la exposición al humo de cigarrillo. 
La importancia de la exposición al humo de segunda y tercera mano se relaciona con la percepción de riesgo; los resultados evidenciaron que entre más cercanía afectiva tiene el fumador a la persona expuesta al humo menor es su percepción del riesgo. Este efecto podría ser explicado desde dos perspectivas: 1) la centrada en la baja percepción de riesgo que tienen los fumadores, y 2) la centrada en la naturaleza social de las creencias, ya que las creencias y comportamientos asociados a la protección en la salud están mediados por la norma subjetiva (i.e. teoría de acción razonada, creencias respecto a lo que el grupo social espera que haga la persona) (Ajzen, 1991; Ajzen y Fishbein, 1973; Cheesman y Suárez, 2014). No obstante, para concluir al respecto, es preciso realizar investigaciones que aborden el efecto del intercambio social en la activación de las creencias o en la exposición directa e indirecta a la sustancia.

\section{Conclusiones}

A partir del modelo que analiza los factores predictores del consumo de cigarrillo es posible afirmar que el consumo de tabaco está determinado, en primer lugar, por factores sociodemográficos como el sexo, la edad y el tiempo que la persona lleva fumando y, en segundo lugar, por las expectativas positivas acerca de los efectos de la sustancia que le proveen funcionalidad al comportamiento que facilitan la activación de los deseos de consumir ante la aparición de señales internas de estrés o desasosiego o de externas de corte social enfocadas a la aceptación por parte del grupo de referentes sociales. En otras palabras, tal como lo habían hallado Castellanos y Londoño (2014) y Rodríguez y Londoño (2010), las expectativas sobre los efectos del cigarrillo conllevan a que estímulos discriminativos motiven al fumador a consumir para conseguir los efectos positivos esperados.

Los hallazgos del presente estudio confirman la existencia de distintos niveles de consumo. El fumador dependiente (con mayor presencia de hombres, aunque las mujeres con tendencia a la igualación) tiene expectativas de consumo relacionadas con efectos fisiológicos que se refuerzan por la provocación de condiciones de dependencia que los obligan a acceder a dosis continuas de la sustancia, condición que se incorpora a sus repertorios conductuales de tal 
modo que hacen parte de su vida. El consumo en este nivel es caracterizado por falta de control de sí mismo, por el hecho de aspirar el humo hasta el pulmón, por combinar consumo de cigarrillo con alcohol y por la dificultad para respetar lugares con prohibición de consumo. El consumidor fuerte tiene expectativas mixtas de recuperar el control y cumplir las expectativas asociadas a las motivaciones sociales, por tanto, su consumo ocurre en ambas condiciones; el consumidor moderado (equilibrado tanto en mujeres como hombres) refiere también preocupación por su estado de salud y expectativas y motivación de consumo de índole social; por último, el consumidor leve estaría motivado a consumir por situaciones sociales más aún si son de celebración.

Es importante anotar que gran parte de los fumadores requieren acompañamiento para abandonar el consumo, tal como lo propone la estrategia MPOWER. Esta estrategia incluye el ofrecimiento de la ayuda necesaria para abandonar el uso del tabaco y requeriría que en Colombia se dirijan los esfuerzos a la creación de clínicas de atención al fumador como estrategia complementaria a la implementación de la Ley antitabaco y las acciones preventivas adelantadas. Los resultados de la presente investigación facilitan la comprensión y el abordaje de la interacción de procesos psicológicos (creencias, tentaciones e identidad), condiciones sociales y la topografía de uso de la sustancia más detallada, condiciones que deben ser abordadas de manera específica en las acciones preventivas y de intervención; existen fumadores que reconocen los riesgos tanto físicos como psicológicos que afectan su salud de forma integral, e incluso refieren haberlos experimentado, y estos tienen mayor probabilidad de incrementar la percepción de vulnerabilidad y susceptibilidad requeridas en el inicio de verdaderos procesos de disminución y abandono del consumo del tabaco.

\section{Referencias}

American Cancer Society. (2014). Datos sobre el uso del tabaco en los niños y adolescentes. recuperado de http://www.cancer.org/espanol/cancer/queesloquecausaelcancer/ tabacoycancer/fragmentado/datos-sobre-el-uso-del-tabaco-en-los-ninos-y-adolescentes-child-and-teen-tobacco-use 
Ajzen, I. y Fishbein, M. (1973). Attitudinal and normative variables as predictors of specific behavior. Journal of Personality and Social Psychology, 27, 41-57.

Ajzen, I. (1991): The theory of planned behavior, Organizational Behavior and Human Decision Processes, 50, 179-211.

Álvarez-Aguirre, A., Alonso-Castillo, M. y Guidorizzi, A. (2014). Factores predictivos del uso de alcohol y tabaco en los adolescentes. Revista Latinoamericana de Enfermagem, 22(6), 1056-1062. doi: https://dx.doi.org/10.1590/01041169.3570 .2516

Ávila, K. (2003). Consumo de sustancias psicoactivas capaces de producir dependencia. San José: Centro de Desarrollo Estratégico e Información en Salud y Seguridad Social (CENDEISSS).

Aveyard P. y West, R. (2007). Managing smoking cessation. Clinical Review BMJ, 335-337 doi: https://doi.org/10.1136/bmj.39252.591806.47

Baha, M. y Le Faou, A. (2013). Perceived risks to smoking cessation among treatment-seeking French light smokers. Preventive Medicine, 57, 372-376. doi: 10.1016/j.ypmed.2013.06.020

Bautista, F., Gómez, V., Aguilar, A., Herrador, F. y Alfaro, A. (2016). Estudio de prevalencia del consumo de tabaco en estudiantes universitarios. Primera edición. San Salvador, El Salvador: UEEs Editorial.

Becoña, E. y Vázquez, F. (1997). La evaluación de la conducta de fumar. Psicología Conductual, 5(3), 345-364. Recuperado de http://www.psicologiaconductual. com/PDFespanol/1997/art08.3.05.pdf

Becoña, E. (2002). Bases cientificas de la prevención de las drogodependencias. Madrid: Ministerio del Interior, delegación del gobierno para el plan nacional sobre drogas secretaría general técnica.

Becoña, E. y Lorenzo, M. (2014). Evaluación de la conducta de fumar. Adicciones, 16, 201-226.

Becoña, E., Arias, F., Barreiro, C., Berdullas, J., Iraurgui, I., Llorente, J., López, A. et al. (2011). Manual de adicciones para psicólogos especialistas en psicología clínica en formación. Barcelona: Socidrogalcohol.

Bisquerra, R. (1989). Métodos de investigación educativa: Guía práctica. Barcelona: CEAC.

Borracci R. y Mulassi A. (2015). Tobacco use during adolescence may predict smoking during adulthood: simulation-based research. Arch Argent Pediatr. 113(2), 106-112. doi: 10.5546/aap.2015.106. 
Brown, A., Carpenter, M. y Sutfin, E. (2011). Occasional smoking in college: Who, what, when and why? Addictive Behaviors, 36, 1199-1204. doi: 10.1016/j.addbeh.2011.07.024

Calleja, N. (2012). Susceptibilidad al consumo de tabaco y comportamiento tabáquico en las adolescentes. Universitas Psychologica, 11(4), 1227-1234.

Castellanos, P. y Londoño, C. (2014). Identidad, percepción del riesgo y consumo de cigarrillo en adolescentes universitarios (Tesis de Maestría). Recuperada de: http://hdl.handle.net/10983/2083

Castañal-Canto, X., Martín-Miguel, M., Hervés-Beloso, C., Pérez-Cachafeiro, S., Espinosa-Arévalo, M. y Delgado-Martín, J. (2012). Trabajando con nuestros pacientes fumadores en atención primaria. Un análisis de coste-efectividad. Atención Primaria, 44(11), 659-666.

Campo-Arias, A. y Díaz-Martínez, L. (2006). Prevalencia y factores asociados con el consumo diario de cigarrillo en mujeres adultas de Bucaramanga, Colombia. Revista Colombiana de Obstetricia y Ginecología, 57(4), 236-244.

Centro para el Control y la Prevención de Enfermedades (2014). Consejos de exfumadores. Recuperado de: http://www.cdc.gov/tobacco/campaign/tips/spanish/

Cheesman, S., y Suárez, N. (2014). Risk and protective factors of smoking among students of Medical Sciences in the Science Faculty usac, Guatemala. Horizonte sanitario, 13(3), 223-232.

Clemente, M., Moreno, C., Rubio, E., Cantera, C., Puente, D. y Sierra, N. (2012). Actitudes de los españoles frente a los espacios libres de humo de tabaco. Atención Primaria, 44(3), 138-144. doi: 10.1016/j.aprim.2011.01.015

Cogollo, Z. y Gómez, E. (2013). Asociación entre consumo de cigarrillo, alcohol y sustancias ilegales en adolescentes estudiantes en Cartagena, Colombia. Revista Hacia la Promoción de la Salud, 8(1), 110-117.

Comunidad Andina (2013). II Estudio Epidemiológico Andino sobre Consumo de Drogas en la Población Universitaria. Informe Regional, 2012. Recuperado de: http://www.cicad.oas.org/oid/pubs/PRADICAN_Informe_Regional.pdf

Córdoba, R. y Samitier, E. (2015). 50 mitos del tabaco. Departamento de Salud y Consumo: Gobierno de Aragón. Recuperado de: http://www.cnpt.es/doc_pdf/ libro_50_mitos_tabaco.pdf

Departamento Administrativo Nacional de Estadística DANE (2016). Certificado de precios de cigarrillos para el primer semestre del año 2015. 
Departamento Administrativo Nacional de Estadística DANE (2020). Estratificación. Recuperado de: https://www.dane.gov.co/index.php/servicios-al-ciudadano/ servicios-informacion/estratificacion-socioeconomica\#normatividad

DeLay, D., Kiuru, N., Salmela-Aro, K. y Nurmi, J. (2013). Selecting and Retaining Friends on the Basis of Cigarette Smoking Similarity. Journal Research on Adolescence, 23(3), 464-473. doi: 10.1111/jora.12017

Ferreira, E., Sivalli, C. y Baldini, C. (2014). Contextos de inicio do consumo de tabaco em diferentes grupos sociais. Revista Latino-americana de Enfermagem, 22(3), 379-385. doi: 10.1590/0104-1169.3205.2427

García del Castillo, J. (2012). Concepto de percepción de riesgo y su repercusión en las adicciones. Salud y drogas, 12, 133-151.

Gobierno Nacional de la República de Colombia. (2013). Estudio Nacional de Consumo de Sustancias Psicoactivas en Colombia - 2013, informe final. Recuperado de: https://www.unodc.org/documents/colombia/2014/Julio/Estudio_de_Consumo_UNODC.pdf

Haight, J., Dickter, C. y Forestell, C. (2012). A comparison of daily and occasional smokers' implicit affective responses to smoking cues. Addictive Behaviors, 37, 234-239. doi: 10.1016/j.addbeh.2011.10.006

Halterman, J., Borrelli, B., Conn, K., Tremblay P. y Blaakman, S. (2010). Motivation to quit smoking among parents of urban children with asthma. Patient Education and Counseling, 79, 152-155. doi: 10.1016/j.pec.2009.09.004

Hanson, M. (2014). Beliefs About Smoking in Millennial Generation Teenage Women. The Journal for Nurse Practitioner, 10(3) 162-166.

Li, X., Mao, R., Stanton, B. y Qun, Z. (2010). Parental, Behavioral, and Psychological Factors Associated with Cigarette Smoking among Secondary School Students in Nanjing, China. Journal of Child \& Family Studies, 19(3), 308-317. doi: $10.1007 / s 10826-009-9299-1$

Londoño, C., y Rodríguez, I. (2007). Cuestionario de expectativas hacia el cigarrillo (CEC documento de trabajo). Universidad Católica de Colombia.

Londońo, C., Cabarcas, K. y Velasco, M. (2020). Programa de prevención indicada para la cesación del consumo de cigarrillo "Libre de Tabaco Px4". Acciones basadas en evidencia científica. Logos Vestogium, 7. Universidad Católica de Colombia.

Londoño, C., Velasco, M., Pardo, C., López, A., López, Y., Mosquera, Y., Tapias, L. et al., (en prensa). Validación de la Batería de Evaluación de Aspectos Psicoló- 
gicos Asociados al Consumo de Cigarrillo (BEPSICC) en Adolescentes. Proyecto de investigación docente. Facultad de Psicología. Universidad Católica de Colombia

Lujic, C., Reuter, M. y Netter, P. (2005). Psychobiological theories of smoking and smoking motivation. European Psychologist. 10 (1) 1-24.

Marqueta, A., Nerín, I., Jiménez-Muro, A., Gargallo P. y Beamonte, A. (2013). Predictors of outcome of a smoking cessation treatment by gender. Gaceta Sanitaria, 27, 26-31. doi: http://dx.doi.org/10.1016/j.gaceta.2011.12.011

Ministerio de Salud y Protección Social (2015). Encuesta Nacional de Salud Mental. Bogotá, Colombia.

Molano, N. y Montua, A. (2015). Perception of cigarette smoking among youth in the University of Cauca. Revista Impetus, 9(1), 59-64

Novoa-Gómez, M., Barreto, I. y Silva, L. (2012). Consumo de cigarrillo y prácticas culturales en contextos universitarios. Revista Latinoamericana de Psicología. 44, 97-110.

Organización Mundial de la Salud (2020). La mujer y el tabaco: atracción fatal. Boletín de la Organización Mundial de la Salud, 88, 561-640.

Organización Panamericana de la Salud (2014). Informe sobre control de tabaco para la región de las Américas. Washington, DC: OPs.

Ortiz, Y. y Rodríguez, J. (2009). Prevalencia y características del consumo de tabaco en adolescentes del Colegio Universitario Santiago de Cali. Revista Facultad de Medicina de la Universidad Nacional de Colombia, 57(2), 89-99.

Rodríguez, I. y Londoño, C. (2010). El proceso de adopción de precauciones en la prevención secundaria del consumo de cigarrillo en estudiantes universitarios. Acta Colombiana de Psicología, 13(1), 79-90.

Rubinstein, M., Rait, M., Sen, S. y Shiffman, S. (2014). Characteristics of adolescent intermittent and daily smokers. Addictive Behaviors, 39, 1337-1341. doi: http:// dx.doi.org/10.1016/j.addbeh.2014.04.021

Sandoyal, E. y Bianco, E. (2011). Mortalidad por tabaquismo y por humo de segunda mano en Uruguay. Revista Uruguaya de Cardiología, 26(3), 201-206.

Velasco, M., Londońo, C., Forero, M., Paéz, D., Romero, M. y Ruiz, C. (2017). Identidad de consumo, motivos y creencias en jóvenes fumadores y no fumadores colombianos. Drugs and Addictive Behavior, 2(2), 170-192. doi: https://doi. org/10.21501/24631779.2439 
Villena, A., Morena, S., Párraga, I., González, M., Soriano, H. y López-Torres, J. (2009). Factores asociados al consumo de tabaco en adolescentes. Revista Clínica de Medicina de Familia, 2(7), 320-325.

Wood, C., Cano-Vindel, A., Iruarrizaga, I., Dongil, E., Salguero, J. (2010). Relaciones entre estrés, tabaco y trastorno de pánico. Ansiedad y Estrés. 16(2-3), 309-325. 